Quantum Interference in the Control of Molecular Processes Author(s): P. Brumer and M. Shapiro

Source: Philosophical Transactions: Mathematical, Physical and Engineering Sciences, Vol. 355, No. 1733, Highlights in Quantum Optics (Dec. 15, 1997), pp. 2409-2412

Published by: The Royal Society

Stable URL: http://www.jstor.org/stable/54947

Accessed: 06/09/2008 16:38

Your use of the JSTOR archive indicates your acceptance of JSTOR's Terms and Conditions of Use, available at http://www.jstor.org/page/info/about/policies/terms.jsp. JSTOR's Terms and Conditions of Use provides, in part, that unless you have obtained prior permission, you may not download an entire issue of a journal or multiple copies of articles, and you may use content in the JSTOR archive only for your personal, non-commercial use.

Please contact the publisher regarding any further use of this work. Publisher contact information may be obtained at http://www.jstor.org/action/showPublisher?publisherCode=rsl.

Each copy of any part of a JSTOR transmission must contain the same copyright notice that appears on the screen or printed page of such transmission.

JSTOR is a not-for-profit organization founded in 1995 to build trusted digital archives for scholarship. We work with the scholarly community to preserve their work and the materials they rely upon, and to build a common research platform that promotes the discovery and use of these resources. For more information about JSTOR, please contact support@jstor.org. 


\title{
Quantum interference in the control of molecular processes
}

\author{
BY P. BRUMER ${ }^{1}$ AND M. SHAPIRO ${ }^{2}$ \\ ${ }^{1}$ Chemical Physics Theory Group and The Ontario Laser and Lightwave Research \\ Centre, University of Toronto, Toronto, Canada M5S $1 A 1$ \\ ${ }^{2}$ Chemical Physics Department, Weizmann Institute of Science, \\ Rehovot, Israel 76100
}

Coherent control of molecular processes provides a means of controlling the dynamics of molecules, and of chemical reactions, via laser induced quantum interference. We briefly introduce this approach, provide relevant references and note two recent exciting advances in the control of chemical processes.

Many papers presented at this Discussion Meeting share a common theme in that they describe quantum optics applications which take advantage of coherence properties of matter and radiation. Here we call the attention of the quantum optics community to a parallel development of coherence-based ideas in chemical physics. In particular, since 1986, efforts to control molecular motion and molecular proceses have turned to the use of quantum interference as a means of directing molecular motion towards desired goals. Below we provide a brief sketch of the ideas which underly this work and call attention to two particularly exciting developments in this area. Both comprehensive (Shapiro \& Brumer 1997) and elementary reviews (Brumer \& Shapiro 1995) are available elsewhere.

To introduce the essence of coherent control, consider a chemical reaction which, at total energy $E$, produces a number of distinct products. The total Hamiltonian is denoted $H=H_{q}^{0}+V_{q}$, where $H_{q}^{0}$ is the Hamiltonian of the separated products in the arrangement channel labelled by $q(q=1,2, \ldots)$ and $V_{q}$ is the interaction between products in arrangement $q$. For example, $q=1,2$ may be the $\mathrm{A}+\mathrm{BC}$ and $\mathrm{AB}+\mathrm{C}$ products, respectively, of the photon induced dissociation of a molecule denoted ABC,

$$
\mathrm{A}+\mathrm{BC} \leftarrow \mathrm{ABC} \rightarrow \mathrm{AB}+\mathrm{C} .
$$

We denote eigenvalues of $H_{0}^{q}$ by $\left|E, n, q^{0}\right\rangle$, where $n$ denotes all quantum numbers other than $E$. Eigenfunctions of $H$, which correlate with $\left|E, n, q^{0}\right\rangle$ at large product separation, are labelled $\left|E, n, q^{-}\right\rangle$. By the definition (see, for example, Taylor 1972) of $\left|E, n, q^{-}\right\rangle$, a state prepared experimentally as a superposition $|\Psi(t=0)\rangle=$ $\sum_{n, q} c_{n, q}\left|E, n, q^{-}\right\rangle$has probability $\left|c_{n, q}\right|^{2}$ of forming product in channel $q$, with quantum numbers $n$. As a consequence, the probability of forming a product in any asymptotic state is equal to the probability of initially forming the appropriate minus state which correlates with the desired product. The essence of control lies, therefore, in forming the desired linear combination at the time of preparation. The essence of the coherent radiative control approach is to utilize phase and intensity properties of laser 
excitation to alter the character of the prepared state so as to enhance production of the desired product.

Many of the proposed coherent control scenarios rely upon a simple way of achieving active control over the prepared state and product. Specifically, active control over products is achieved by driving an initially pure molecular state through two or more independent coherent optical excitation routes. The resultant product probability displays interference terms between these two routes, whose magnitude and sign depend upon laboratory parameters. As a consequence, product probabilities can be manipulated directly in the laboratory.

This approach has a well-known analogy, the interference between paths as a beam of either particles or of light passes through a double slit. In that instance, source coherence leads to either constructive or destructive interference, manifest as patterns of enhanced or reduced probabilities on an observation screen. In the case of coherent control, the overall coherence of a pure state plus laser source allows for the constructive or destructive manipulation of probabilities in product channels.

Consider, as an example of coherent control, a specific scenario for unimolecular photoexcitation (Shapiro et al. 1988; Chan et al. 1991) where a system, initially in pure state $\left|E_{i}\right\rangle$, is excited to energy $E$, by simultaneous application of a CW field and its third harmonic,

$$
\epsilon(t)=\epsilon_{3} \hat{\boldsymbol{\epsilon}}_{3} \cos \left[\left(\omega_{3}+\theta_{3}\right) t\right]+\epsilon_{1} \hat{\boldsymbol{\epsilon}}_{1} \cos \left[\left(\omega_{1}+\theta_{1}\right) t\right]
$$

$\left(\omega_{3}=3 \omega_{1}\right)$, providing two independent optically driven routes from $\left|E_{i}\right\rangle$ to $\left|E, n, q^{-}\right\rangle$. Here, $\hat{\epsilon}_{i}(i=1,3)$ denotes a unit vector in the $i$ th field direction.

Straightforward perturbation theory, valid for the weak fields under consideration, gives the probability $P\left(E, q ; E_{i}\right)$ of forming product at energy $E$ in arrangement $q$ as

$$
P\left(E, q ; E_{i}\right)=P_{3}\left(E, q ; E_{i}\right)+P_{1}\left(E, q ; E_{i}\right)+P_{13}\left(E, q ; E_{i}\right) .
$$

Here, $P_{3}\left(E, q ; E_{i}\right)$ is the probability arising from the one photon route,

$$
P_{3}\left(E, q ; E_{i}\right)=\left(\frac{\pi}{\hbar}\right)^{2} \epsilon_{3}^{2} \sum_{n}\left|\left\langle E, n, q^{-}\left|\left(\hat{\boldsymbol{\epsilon}}_{3} \cdot \boldsymbol{\mu}\right)_{e, g}\right| E_{i}\right\rangle\right|^{2},
$$

where $\boldsymbol{\mu}$ is the electric dipole operator and $\left(\hat{\boldsymbol{\epsilon}}_{3} \cdot \boldsymbol{\mu}\right)_{e, g}=\left\langle e\left|\hat{\boldsymbol{\epsilon}}_{3} \cdot \boldsymbol{\mu}\right| g\right\rangle$, where $|g\rangle$ and $|e\rangle$ are the ground and excited electronic state wave functions, respectively. The second term is the photodissociation contribution from the three photon route given by

$$
P_{1}\left(E, q ; E_{i}\right)=\left(\frac{\pi}{\hbar}\right)^{2} \epsilon_{1}^{6} \sum_{n}\left|\left\langle E, n, q^{-}|T| E_{i}\right\rangle\right|^{2},
$$

with

$$
T=\left(\hat{\boldsymbol{\epsilon}}_{1} \cdot \boldsymbol{\mu}\right)_{e, g}\left(E_{i}-H_{g}+2 \hbar \omega_{1}\right)^{-1}\left(\hat{\boldsymbol{\epsilon}}_{1} \cdot \boldsymbol{\mu}\right)_{g, e}\left(E_{i}-H_{e}+\hbar \omega_{1}\right)^{-1}\left(\hat{\boldsymbol{\epsilon}}_{1} \cdot \boldsymbol{\mu}\right)_{e, g} .
$$

The final and most significant term, $P_{13}\left(E, q ; E_{i}\right)$, arises from one photon-three photon interference,

$$
P_{13}\left(E, q ; E_{i}\right)=-2(\pi / \hbar)^{2} \epsilon_{3} \epsilon_{1}^{3} \cos \left(\theta_{3}-3 \theta_{1}+\delta_{13}^{(q)}\right)\left|F_{13}^{(q)}\right|,
$$

with the amplitude $\left|F_{13}^{(q)}\right|$ and phase $\delta_{13}^{(q)}$ defined by

$$
\left|F_{13}^{(q)}\right| \exp \left(\mathrm{i} \delta_{13}^{(q)}\right)=\sum_{n}\left\langle E_{i}|T| E, n, q^{-}\right\rangle\left\langle E, n, q^{-}\left|\left(\hat{\boldsymbol{\epsilon}}_{3} \cdot \boldsymbol{\mu}\right)_{e, g}\right| E_{i}\right\rangle .
$$


The branching ratio $R_{q q^{\prime}}$ for channels $q$ and $q^{\prime}$ can then be written as

$$
R_{q q^{\prime}}=\frac{P\left(E, q ; E_{i}\right)}{P\left(E, q^{\prime} ; E_{i}\right)}=\frac{F_{3}^{(q)}-2 x \cos \left(\theta_{3}-3 \theta_{1}+\delta_{13}^{(q)}\right) \epsilon_{0}^{2}\left|F_{13}^{(q)}\right|+x^{2} \epsilon_{0}^{4} F_{1}^{(q)}}{F_{3}^{\left(q^{\prime}\right)}-2 x \cos \left(\theta_{3}-3 \theta_{1}+\delta_{13}^{\left(q^{\prime}\right)}\right) \epsilon_{0}^{2}\left|F_{13}^{\left(q^{\prime}\right)}\right|+x^{2} \epsilon_{0}^{4} F_{1}^{\left(q^{\prime}\right)}},
$$

where

$$
F_{3}^{(q)}=\left(\frac{\hbar}{\pi}\right)^{2} \frac{P_{3}\left(E, q ; E_{i}\right)}{\epsilon_{3}^{2}}, \quad F_{1}^{(q)}=\left(\frac{\hbar}{\pi}\right)^{2} \frac{P_{1}\left(E, q ; E_{i}\right)}{\epsilon_{1}^{6}},
$$

with $F_{3}^{\left(q^{\prime}\right)}$ and $F_{1}^{\left(q^{\prime}\right)}$ defined similarly. Here, $x=\bar{\epsilon}_{1}^{3} / \bar{\epsilon}_{3}$ with $\epsilon_{l}=\bar{\epsilon}_{l} \epsilon_{0}$; the quantity $\epsilon_{0}$ essentially carries the unit for the electric field.

The numerator and denominator of equation (1.8) each display what we regard as the canonical form for coherent control. That is, independent contributions from more than one route which are modulated by an interference term. Since the interference term is controllable through variation of laboratory parameters (here the relative intensity and relative phase of the two lasers), so too is the product ratio $R$.

This scenario has been investigated both computationally (Chan et al. 1991) and experimentally (Chen et al. 1990; Chen \& Elliott 1990; Park et al. 1991; Zhu et al. 1995). Both show that extensive control over product probabilities is possible.

Once one appreciates the essence of coherent control, i.e. the simultaneous coherent excitation of the system by multiple routes, numerous scenarios can be devised (Shapiro \& Brumer 1997), many of which have been computationally shown to provide highly successful control schemes. Here we call attention to one particularly exciting prospect (Chen et al. 1995) in which a bound state of a molecular system is excited to the continuum by a laser of frequency $\omega_{1}$ which is, in turn, coupled to another (initially empty) bound state by a strong laser of frequency $\omega_{2}$. Varying either frequency can be shown to provide an effective means of controlling the ratio of photodissociation products. This method, which we call Incoherent Interference Control, does not require lasers which are relatively coherent.

Both experimental and theoretical studies of Incoherent Interference Control (Chen et al. 1995; Shnitman et al. 1996) show it to be a very effective means of controlling the dynamics of photodissociation. In particular, a recent study of the dissociation of $\mathrm{Na}_{2}$ to produce different atomic products showed that one could significantly increase the production of $\mathrm{Na}(3 \mathrm{~s})+\mathrm{Na}(3 \mathrm{p})$ while simultaneously reducing the production of $\mathrm{Na}(3 \mathrm{~s})+\mathrm{Na}(3 \mathrm{~d})$ by varying $\omega_{2}$ over $3 \mathrm{~cm}^{-1}$. Experiment and theory were found to be in excellent agreement. Most recently we have examined the possibility of improving control over cross-sections by varying pulse orderings, intensities and widths in a pulsed laser version of incoherent interference control. Our results showed that excellent control over cross-sections is possible for a wide range of laser pulse parameters (Shapiro et al. 1997).

Finally we note that the vast majority of work in coherent control has focused on unimolecular processes, i.e. processes involving excitation of a single molecule, such as that in equation (1.1). However, the vast majority of chemical reactions of interest are bimolecular in nature, i.e. of the type

$$
\mathrm{A}+\mathrm{B} \rightarrow \mathrm{C}+\mathrm{D}
$$

Attempts to control such processes have been limited by the difficulty of preparing a superposition of controllable degenerate collisional eigenstates. Our recent work (Shapiro \& Brumer 1996) suggests an approach to solving this problem and opens up a vast new range of applications.

Phil. Trans. R. Soc. Lond. A (1997) 
This work was supported by the US Office of Naval Research.

\section{References}

Brumer, P. \& Shapiro, M. 1995 Laser control of chemical reactions. Scientific Am. 272, 56-63.

Chan, C. K., Brumer, P. \& Shapiro, M. 1991 Coherent radiative control of IBr photodissociation via simultaneous $\left(\omega_{1}, \omega_{3}\right)$ excitation. J. Chem. Phys. 94, 2688-2696.

Chen, C. \& Elliott, D. S. 1990 Measurements of optical phase variations using interfering multiphoton ionization processes. Phys. Rev. Lett. 65, 1737-1740.

Chen, C., Yin, Y.-Y. \& Elliott, D. S. 1990 Interference between optical transitions. Phys. Rev. Lett. 64, 507-510.

Chen, Z., Shapiro, M. \& Brumer, P. 1995 Incoherent interference control of two-photon dissociation. Phys. Rev. A 52, 2225-2233.

Park, S. M., Lu, S.-P. \& Gordon, R. J. 1991 Coherent laser control of the resonance-enhanced multiphoton ionization of HCl. J. Chem. Phys. 94, 8622-8624.

Shapiro, M. \& Brumer, P. 1996 Coherent control of collisional events: bimolecular reactive scattering. Phys. Rev. Lett. 77, 2574-2576.

Shapiro, M. \& Brumer, P. 1997 Quantum control of chemical reactions. Trans. Faraday Soc. 93, $1263-1277$.

Shapiro, M., Hepburn, J. \& Brumer, P. 1988 Simplified laser control of unimolecular reactions: simultaneous $\left(\omega_{1}, \omega_{3}\right)$ excitation. Chem. Phys. Lett. 149, 451-454.

Shapiro, M., Chen, Z. \& Brumer, P. 1997 Simultaneous control of selectivity and yield of molecular dissociation: pulsed incoherent interference control. Chem. Phys. 217, 325-340.

Shnitman, A., Sofer, I., Golub, I., Yogev, A., Shapiro, M., Chen, Z. \& Brumer, P. 1996 Experimental observation of laser control: electronic branching in the photodissociation of $\mathrm{Na}_{2}$. Phys. Rev. Lett. 76, 2886-2889.

Taylor, J. R. 1972 Scattering theory. John Wiley.

Zhu, L., Kleiman, V., Li, X., Lu, S.-P., Trentelman, K. \& Gordon, R. J. 1995 Coherent laser control of the product distribution obtained in the photoexcitation of HI. Science $\mathbf{2 7 0}, 77-80$. 